\title{
The renaissance of the macrolides: New and changing roles in infectious diseases
}

\author{
LIONEL A MANDELL, MD, FRCPC
}

$I^{\prime}$ N DEALING WITH ANY INFECTION, WE MUST CONSIDER THREE variables which together form an interactive triad. These include the host, the pathogen and the drug(s) used in treatment. This is not a static relationship but is in fact a rather dynamic one, and we have seen changes over the years in each of these three variables. As for the host, patients may have been previously well or may have comorbid conditions which may include a variety of maladies from simple congestive heart failure to metastatic neoplastic disease. From the point of view of the pathogen, relatively new organisms such as Chlamydia pneumoniae may be encountered, or organisms may become more virulent as has occurred with the Group A streptococcus or they may develop resistance to previously used drug therapy. Some disturbing examples of this are the resistant pneumococcal isolates found in countries such as Spain and Hungary, and multiresistant tuberculosis seen recently in the United States $(1,2)$. From the perspective of drug therapy, a variety of considerations come into play including in vitro activity, pharmacokinetics, efficacy, toxicity and cost.

\section{OLDER MACROLIDES}

The last few decades have witnessed a virtual explosion in the number of antimicrobials available. We have seen the development of newer and more potent beta- lactams in the form of extended spectrum penicillins, third generation cephalosporins and carbapenems. More recently, the fluoroquinolones have captured our attention with an ever increasing number of agents with broader spectra of activity. The macrolides, however, have been with us for almost 40 years, but recently there has been a resurgence of interest in these agents.

Erythromycin, the first of the macrolides, was isolated in 1952 from a strain of Streptomyces erythreus found in a soil sample from the city of Ilo-Ilo in the Philippines (3). Its initial use was primarily against Gram-positive coccal infections, particularly in patients allergic to penicillin, but in the 1960s its activity against Mycoplasma pneumoniae was documented $(4,5)$.

Erythromycin consists of a 14-membered macrocyclic lactone ring to which are attached two sugar moieties; desosamine and cladinose. Following oral ingestion, it breaks down under acidic conditions to 8,9-anhydroerythromycin-6,9-hemiketal and then to its 6,9,9,12-spiroketal form (6). Neither of these acidcatalyzed degradation products possesses significant antibacterial activity.

In order to minimize breakdown of the drug by gastric acid and to enhance absorption, a number of oral preparations of erythromycin have been made available. These depend primarily on the use of acidstable enteric coatings or the use of highly water insol-

Division of Infectious Diseases, McMaster University, Hamilton, Ontario

Correspondence: Dr Lionel A Mandell, McMaster Medical Unit, Henderson General Hospital, 711 Concession Street, Hamilton, Ontario LSV 1 C3 
TABLE 1

In vitro activity of macrolides against selected respiratory pathogens - minimum inhibitory concentrations (MIC 90 ) in $\mu \mathrm{g} / \mathrm{mL}$

\begin{tabular}{|c|c|c|c|c|c|}
\hline Organism & Erythromycin & Clarithromycin & Roxithromycin & Azithromycin & Josamycin \\
\hline Streptococcus pneumoniae & 0.03 & 0.015 & 0.03 & 0.12 & 0.12 \\
\hline Haemophilus influenzae & 4 & $1^{*}$ & 8 & 0.5 & 16 \\
\hline Moraxella catarrhalis & 0.25 & 0.25 & 1 & 0.06 & 1 \\
\hline Legionella pneumophila & 2 & 0.25 & 0,5 & 2 & 1 \\
\hline Chlamydia pneumoniae & 0.06 & 0.007 & 0.25 & 0.5 & 0.25 \\
\hline Mycoplasma pneumoniae & 0.004 & 0.031 & - & 0.001 & - \\
\hline
\end{tabular}

${ }^{*}$ Combined activity of clarithromycin and its $14-\mathrm{OH}$ metabolite

uble salts such as lauryl sulfate or stearate. They include enteric-coated and film-coated tablets, entericcoated pellets and capsules and stearate salts prepared as film-coated tablets and esters.

\section{NEW MACROLIDES}

In addition to the above mentioned modifications used to protect erythromycin against acid degradation, a number of additional approaches have been used in order to prevent acid-mediated breakdown. This has led to the development of a number of newer macrolides (eg, clarithromycin, azithromycin, roxithromycin) with altered pharmacokinetic and, in some cases, improved antimicrobial properties. The production of many of these newer compounds came about by modification of the functional groups which took part in the acid-catalyzed degradation reaction. These include the ketone at C-9, the hydroxyl group at C-6, the proton at C-8 and the diol moiety at C-11 and C-12 (6).

These newer compounds contain either a 14-,15- or 16-membered ring structure. The larger 15-membered ring, which forms the basis for the new macrolide azithromycin, was made by inserting a nitrogen into the lactone ring. This modification contributes to the basicity of the molecule as well as to its activity against Gram-negative bacteria (7). A number of 16-membered compounds are under development as well with the major research thrust directed toward acylation of the hydroxyl groups on the lactone ring and the neutral sugar. Such structural changes provide enhanced activity against some resistant organisms and improve oral bioavailability (8).

\section{IN VITRO ACTIVITY}

The antimicrobial mechanism of action of the macrolides is based upon their ability to interfere with protein synthesis by the microorganisms. They accomplish this by binding to the 50 s component of the 70 S ribosome subunit which in turn prevents translocation reactions of the component amino acids required for the building of any proteins (9).

As with virtually any antimicrobial agent, resistance among various pathogens may develop. Resistance to the macrolide class of compounds can be both inducible or expressed constitutively and may be on the basis of chromosomal or plasmid-mediated mechanisms (10). The resistance has a rather distinctive name, being referred to as macrolide, lincosamides, streptogramin B (MLS) resistance, and is ultimately due to the inability of the drug to attach to its binding site due to methylation of residues in the 23SrRNA (10).

If we examine the minimum inhibitory concentrations (MIC90) for the various 14-, 15-, and 16-membered macrolides against Gram-positive cocci such as methicillin-resistant Staphylococcus aureus and Staphylococcus epidermidis, there are virtually no significant differences between these compounds (7). For methicillin-susceptible $S$ aureus, while the range of MICs is the same, the 16-membered compounds appear to have lower MIC90s. Similarly, these compounds all do very well against various streptococcal pathogens, with the 14-membered agents having slightly lower MIC9os than the 15- and 16-membered macrolides (7).

The main concern, however, is with the respiratory pathogens, both the older as well as the newer ones. The main organisms of interest include Streptococcus pneumoniae, Haemophilus influenzae, Moraxella catarrhalis, Legionella species, Chlamydia pneumoniae and $M$ pneumoniae. The in vitro activity of erythromycin and some of the newer macrolides against these pathogens is given in Table 1. All of the agents exhibit excellent activity against $S$ pneumoniae and $M$ catarrhalis. Against $H$ influenzae, it would appear that azithromycin is the most active. In the case of clarithromycin, a unique situation exists. In humans, the major metabolite of clarithromycin is 14-hydroxy clarithromycin. This compound is generally as active as clarithromycin against most pathogens responsible for community-acquired respiratory infections, and two-fold more active than the parent compound against $H$ influenzae $(7,11)$. In testing the two agents together against $H$ influenzae, MICs were usually four- to eight-fold lower for the combination, with a fractional inhibitory concentration (FIC) index ranging from 0.51 to 1.0 (mean FIC index of 0.65 indicates at least an additive effect in vitro) (12).

Against respiratory pathogens such as $L$ pneumophila, the 14-membered macrolides, clarithromycin and roxithromycin, appear to be superior to erythromycin or the 15- and 16-membered lactones, azithromycin 
and josamycin, respectively. Against $C$ pneumoniae, erythromycin appears to be very active, exceeded only by clarithromycin (7).

The pharmacokinetic parameters are where some of the newer agents distinguish themselves from erythromycin. Erythromycin base given in a dose of $500 \mathrm{mg}$ by mouth yields serum levels of 0.3 to $1.9 \mu \mathrm{g} / \mathrm{mL}$. However, the drug is highly protein bound (over 90\%) and its elimination half-life is 1.5 to $3 \mathrm{~h}$, necessitating qid dosing (13).

Clarithromycin, on the other hand, is rapidly absorbed from the gastrointestinal tract - providing good levels in serum as well as tissue - and, unlike erythromycin, is stable in the presence of gastric acid. The oral bioavailabilities of clarithromycin and azithromycin differ somewhat, being approximately $55 \%$ and $37 \%$, respectively (14). Ingestion with food affects these drugs in different ways. The bioavailability of clarithromycin increases by $25 \%$ while that of azithromycin decreases in the presence of food (14).

The distribution of some of these drugs, particularly clarithromycin and azithromycin, is of interest to both investigators and clinicians. Steady state peak serum concentrations of clarithromycin are 1.0 to $1.5 \mu \mathrm{g} / \mathrm{mL}$ after a $250 \mathrm{mg}$ bid dose and 2.0 to $3.0 \mu \mathrm{g} / \mathrm{mL}$ after a $500 \mathrm{mg}$ bid dose (see page 10A of this issue). Serum concentrations of azithromycin are significantly lower. In one study involving 22 patients, the mean serum concentration $12 \mathrm{~h}$ after a single $500 \mathrm{mg}$ dose of azithromycin was $0.13 \mu \mathrm{g} / \mathrm{mL}$. The serum concentrations were generally sub MIC throughout the study period (15). Both drugs achieve levels in lung tissue that exceed MIC9os of pathogens such as $L$ pneumophila, C pneumoniae and $M$ pneumoniae. Also, both agents achieve levels in polymorphonuclear leukocytes up to 16 and 25 times greater than those in serum for clarithromycin and azithromycin, respectively (16).

\section{CLINICAL ACTIVITY}

Macrolides have been used to treat a variety of infections over the years. Those most frequently treated include respiratory tract infections, skin and soft tissue infections, and gastrointestinal infections.

A detailed discussion of various respiratory tract infections treated with macrolides is beyond the scope of this article. However, infections involving virtually every component of the respiratory tract have been treated with macrolides, usually erythromycin. This

\section{REFERENCES}

1. Klugman KP. Pneumococcal resistance to antibiotics. Clin Microbiol Rev 1990;3:171-96.

2. Centers for Disease Control. National action plan to combat multidrug-resistant tuberculosis; Meeting the challenge of multidrug-resistant tuberculosis: summary of a conference; Management of persons exposed to multidrug-resistant tuberculosis. MMWR 1992;41(No RR-11):1-71. agent does well for infections such as otitis media and sinusitis if $S$ pneumoniae is the causative pathogen, but if $H$ influenzae is involved, erythromycin alone may be insufficient and a second drug is generally required $(17,18)$.

For cases of bacterial pharyngitis/tonsillitis due to $S$ pyogenes, erythromycin eliminates the organism and prevents post infectious sequelae such as rheumatic fever and glomerulonephritis.

For cases of community-acquired pneumonia, erythromycin has been the agent of choice for organisms such as $M$ pneumoniae, $C$ pneumoniae and $L$ pneumophila $(19,20)$. Its main drawback has been its lack of consistent activity against $H$ influenzae, preventing its use as single agent therapy for patients with community-acquired pneumonia in whom $H$ influenzae is a possible pathogen. This is obviously one of the potential advantages of these newer drugs. Their broad spectrum of activity allows their use as monotherapy for selected cases of community-acquired pneumonia.

Macrolides such as erythromycin have also been used for treatment of various skin and soft tissue infections such as erysipelas, cellulitis and impetigo. For certain gastrointestinal infections, for example, those caused by Campylobacter jejuni, erythromycin also has a role (21).

Aside from therapeutic indications, erythromycin has also been used prophylactically. It has been used in penicillin-allergic individuals as a prophylactic agent for bacterial endocarditis; it has also been used for prevention of rheumatic fever and in combination with oral neomycin for prophylaxis in patients undergoing colon surgery.

\section{CHANGING ROLES OF THE MACROLIDES}

With the availability of newer macrolides with an enhanced spectrum of activity, improved pharmacokinetics and reduced adverse effects, the role of the macrolides may well be changing. Newer macrolides now offer the prospect of monotherapy for certain cases of community-acquired pneumonia, even when pathogens such as Hinfluenzae are a concern. There are also a number of other possible uses for these newer agents, particularly clarithromycin, for infections caused by Mycobacterium leprae, Mycobacterium avium, Mycobacterium cheloneae, Helicobacter pylori, cryptosporidia and Borrelia burgdorferi.

3. McGuire JM, Bunch RL, Anderson RC, Boaz HE, Flynn EH, Powell HM, Smith JW. "Ilotycin" a new antibiotic. Antibiotics and Chemotherapy 1952;2:281-3.

4. Arai S, Yoshida K, Izawa A, Kumagai K, Ishida N. Effects of antibiotics on growth of Mycoplasma pneumoniae Mac. J Antibiot (A) 1966;19:118-20.

5. Rasch JR, Mogabgab WJ. Therapeutic effect of erythromycin on Mycoplasma pneumoniae pneumonia. 
Antimicrob Agents Chemother 1966:5:693-9.

6. Kirst HA, Sides GD. New directions for macrolide antibiotics: structural modifications and in vitro activity. Antimicrob Agents Chemother 1989;33:1413-8.

7. Hardy DJ, Hensey DM, Beyer JM, Vojtko C, McDonald EJ, Fernandes PB. Comparative in vitro activities of new 14-, 15-, and 16-membered macrolides. Antimicrob Agents Chemother 1988:32:1710-9.

8. Sakakibara H, Omura S. Chemical modification and structure-activity relationship of macrolides. In: Omura S, ed. Macrolide Antibiotics: Chemistry, Biology and Practice. Orlando, Florida: Academic Press, Inc, 1984: 98-119.

9. Gale EF, Cundliffe EC, Reynolds PE, Richmond MH, Waring MJ. The Molecular Basis of Antibiotic Action, 2nd edn. London: Wiley Interscience, 1981;484-8.

10. Foster TJ. Plasmid-determined resistance to antimicrobial drugs and toxic metal ions in bacteria. Microbiol Rev 1983;47:361-409.

11. Fernandes PB, Ramer N, Rode RA, Frieberg CA. Bioassay for A-56268(TE-031) and identification of its major metabolite, 14-hydroxy-6-0 methylerythromycin. Eur J Clin Microbiol Infect Dis 1988:7:73-6.

12. Chin NX, Ga JW, Neu HC. Clarithromycin and its hydroxy metabolite activity against Gram-positive and Haemophilus isolates. Clarithromycin: a new approach to the treatment of community-acquired infections. 7th Mediterranean Congress of Chemotherapy Abstracts,
1990, p 39.

13. Osono T, Umezawa H. Pharmacokinetics of macrolides, lincosamides and streptogramins. J Antimicrob Chemother 1985;16(Suppl A):151-66.

14. Whitman MS, Tunkel AR. Azithromycin and clarithromycin: overview and comparison with erythromycin. Infect Control Hosp Epidemiol 1992;13:357-68.

15. Baldwin DR, Wise R, Andrews JM, Ashby JP, Honeybourne D. Azithromycin concentrations at the site of pulmonary infection. Eur Respir J 1990;3:886-90.

16. Ishiguro $M$, Kaga $H$, Kohno $\mathrm{S}$, Hagashi $\mathrm{T}$, Yamaguchi $\mathrm{K}$, Hirota M. Penetration of macrolides into human polymorphonuclear leukocytes. J Antimicrob Chemother 1989;24:719-29.

17. Howie VM, Ploussard JH. The "in vivo sensitivity test" bacteriology of middle ear exudate during antimicrobial therapy in otitis media. Pediatrics 1969;44:940-4.

18. Giebink GS, Canafax DM. Antimicrobial treatment of otitis media. Semin Resp Infect 1991;6:85-93.

19. Atmar RL, Greenberg SB. Pneumonia caused by Mycoplasma pneumoniae and the TWAR agent. Semin Resp Infect 1989:4:19-31.

20. Muder RR, Yu VL, Fang GD. Community-acquired Legionnaires' disease. Semin Resp Infect 1989;4:32-9.

21. Salazar-Lindo E, Sack RB, Chea-Woo E, et al. Early treatment with erythromycin of Campylobacter jejuni-associated dysentery in children. J Pediatr 1986:109:355. 


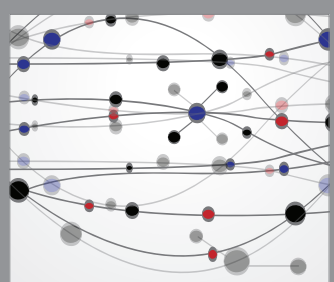

The Scientific World Journal
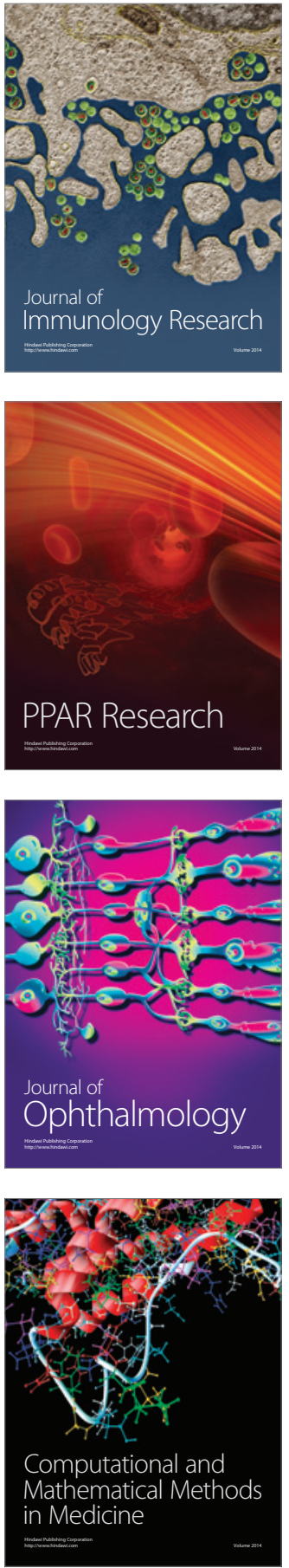

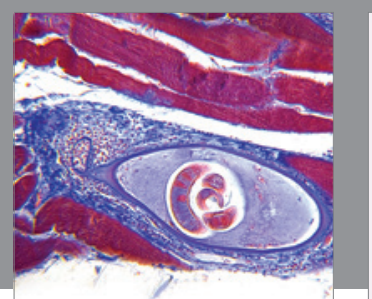

Gastroenterology Research and Practice

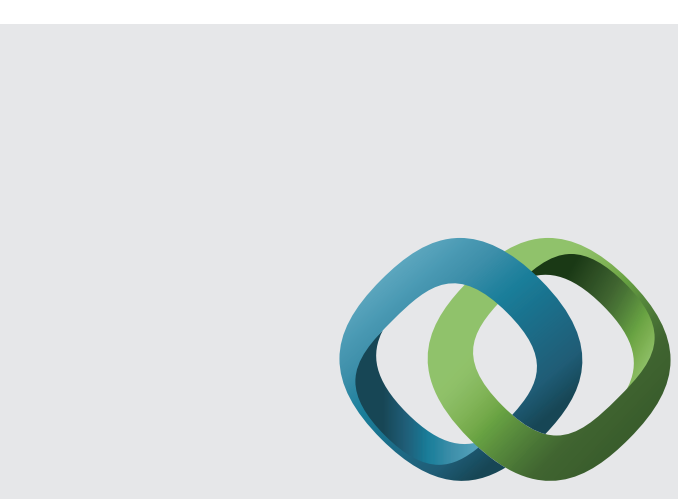

\section{Hindawi}

Submit your manuscripts at

http://www.hindawi.com
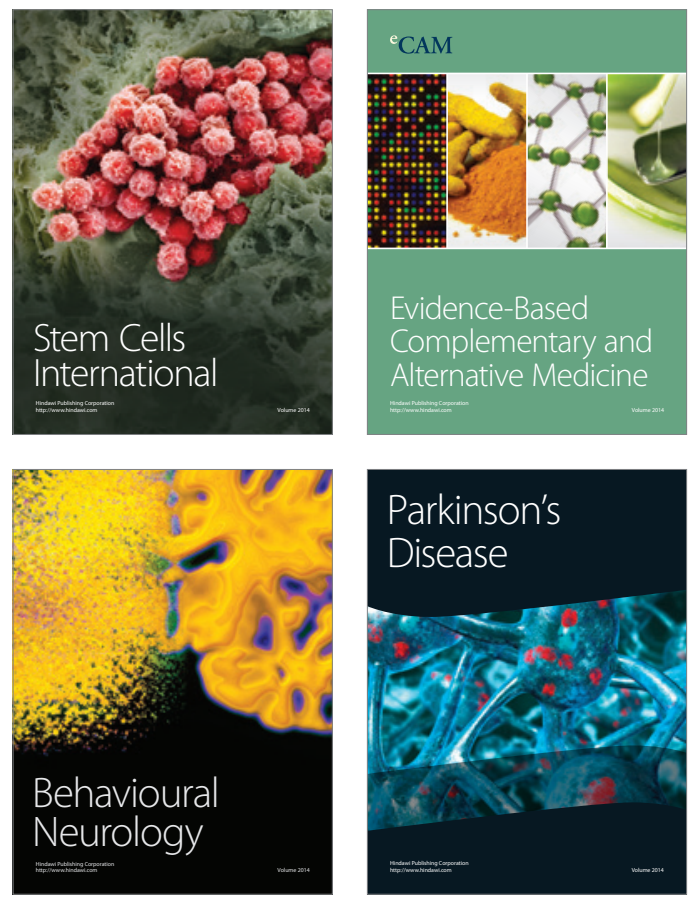
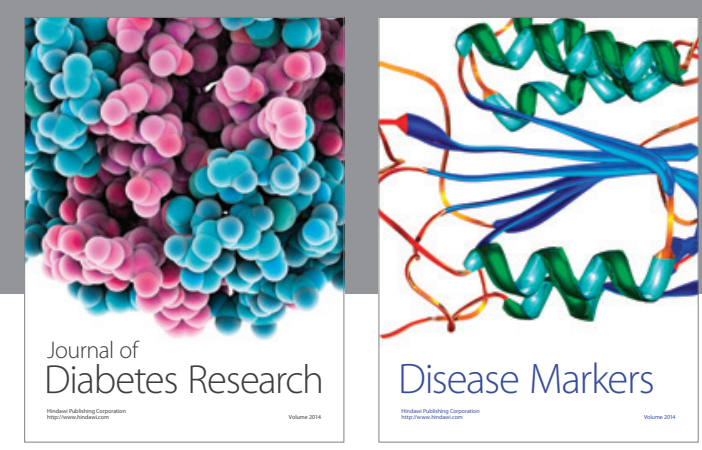

Disease Markers
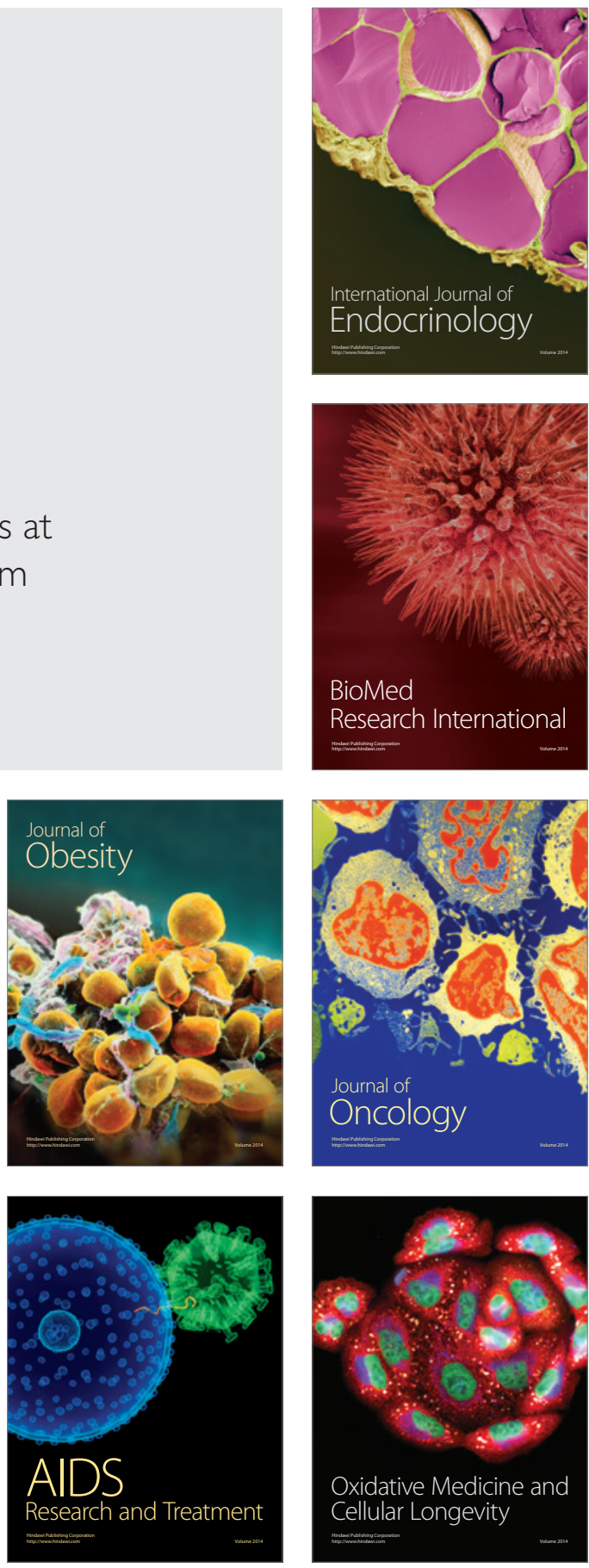